\title{
Details matter: the role of genomic location and assay standardization in DNA methylation analyses
}

\author{
"Only when including quality and standardization at every \\ level of DNA methylation analyses, will we be able to achieve \\ the robustness to independently validate DNA methylation \\ analyses and to compare multiple methylation studies in \\ systematic reviews. This is the only way to more efficiently \\ develop future DNA methylation based biomarkers."
}

First draft submitted: 25 April 2017; Accepted for publication: 25 April 2017; Published online: 15 June 2017

Keywords: biomarkers $\bullet$ design $\bullet$ DNA methylation $\bullet$ epigenetics $\bullet$ genomic location - methodological challenges $\bullet$ normalization $\bullet$ quality $\bullet$ standardization

Close to 60,000 papers mentioning DNA methylation have been published by April 2017, and more than 5000 of them were published last year alone [1]. This amounts to about 14 papers per day demonstrating an exploding interest for performing DNA methylation analyses.

This is not surprising, considering the essential role DNA methylation plays in regulating gene expression [2], impacting essential processes such as cell differentiation. Inactivation of the DNA methyltransferases causes embryonic lethality in mice $[3,4]$, underscoring the importance of correct DNA methylation patterns during normal development, with implications not only in health, but also disease.

Aberrant DNA methylation has been reported in, for example, cardiovascular [5], neurodegenerative [6] and metabolic [7] disease. A link between DNA methylation and cancer was demonstrated as early as 1983 , when a substantial proportion of $\mathrm{CpG}$ sites that were methylated in normal tissues were found to be unmethylated in cancer cells [8]. This hypomethylation, frequently seen early in cancer development, is commonly followed by locus-specific hypermethylation. Large-scale analyses have shown that DNA methylation aberrations are frequently present in cancers [9] and that DNA methylation profiling enables distinguishing cancer subtypes [10] and classifying cancers of unknown primary origin [11].

A variety of DNA methylation aberrations have been suggested as biomarkers for early detection, prognostication and monitoring of cancer, including in noninvasive clinical material such as blood, stool, urine and bile. Great hopes are also tied to DNA methylation as a direct target for epigenetic therapy. Technological advancements, including genome-wide profiling of DNA methylation aberrations in groups of diseased individuals and healthy controls, are supporting a steady increase in the number of DNA methylation based biomarkers, especially for cancer.

However, despite the enormous amount of papers that are being published on DNA methylation, hardly any of these findings enter routine clinical practice. This low success rate can be explained by the observation that the DNA methylation marker field suffers from general methodological issues that affect biomedical and biomarker research, such as insufficiently stringent methodology, low-quality reporting (including lack of adherence to reporting guidelines such as CONSORT, STARD,

\section{Guro E Lind}

Author for correspondence: Department of Molecular Oncology, Institute for Cancer Research \& Colorectal Cancer Research Centre, Oslo University Hospital, Oslo, Norway and

K.G. Jebsen Colorectal Cancer Research Centre, Oslo University Hospital, Oslo, Norway

guro.elisabeth.lind@rr-research.no

\section{Manon van Engeland}

Author for correspondence: Department of Pathology, GROW-School for Oncology \& Developmental Biology, Maastricht University Medical Center, Maastricht, The Netherlands

m.vanengeland@maastrichtuniversity.nl
Future
Medicine $\mathrm{fs}$ par of 
PRISMA, ARRIVE, REMARK), lack of collaborative studies and insufficient independent validation, preregistration, rigorous systematic reviews, umbrella reviews, randomized trials and lack of (de)implementation studies [12].

However, in order to be able to adequately perform some of the steps recently recommended by Ioannidis and Bossuyt [12], such as independent validation and systematic reviews, the specific details of DNA methylation analyses regarding assay design and genomic location should be standardized and adequately reported.

"Close to 60,000 papers mentioning DNA methylation have been published by April 2017, and more than 5000 of them were published last year alone."

One of these details is the variety of locus-specific analyses that are available for analyzing DNA methylation, as reviewed in [13]. The individual methods have various advantages and disadvantages and the choice of method will obviously affect the end results. This is exemplified by a comprehensive review of the analysis of the methylation status of the $M G M T$ promoter [14]. MGMT recently entered clinical guidelines as a predictive biomarker of treatment with alkylating chemotherapy in elderly patients with glioblastoma [15]. In the review, details of all published MGMT studies are summarized, including the choice of locus-specific analysis. The range of methylated high-grade gliomas stretched from less than 20 to more than $90 \%$ [14]. Although, this illustrates how also the choice of technology can impact on the outcome that is measured, it does not imply that individual methods to DNA methylation are not comparable. Indeed, the variation among studies using different methods was not larger than among studies using the same method [14]. In addition, we have also shown that, if carefully aligned and optimized regarding genomic location and sensitivity, multiple DNA methylation assays can yield comparable results [16] and that DNA methylation markers can be validated in independent patient series using independent technologies $[17,18]$.

Another key to the comparability of DNA methylation assays is the genomic location of the locus-specific assays [19]. The majority of locus-specific analyses cover only a limited number of $\mathrm{CpG}$ sites, and the subsequent methylation frequency only reflects the methylation status of the selected region for analysis. The sites selected for DNA methylation analyses should therefore be representative. Already in 1999, Deng and colleagues demonstrated that the methylation of the $M L H 1$ promoter was highly variable, and that only a small and specific region of the promoter invariably correlated with the expected absence of gene expression [20]. Similar examples exist, as addressed in an overview by van Vlodrop et al. [19], including the $M A L$ gene. Using a genome-wide approach searching for novel DNA methylation biomarkers for early detection of colorectal cancer, we were happy to identify $M A L$ which, with a sensitivity in the 80 s and a specificity in the 90s, was one of the most promising biomarkers for colorectal cancers at the time [21]. This accuracy was in stark contrast to a previous study of $M A L$ with a sensitivity of only $6 \%$ in colorectal cancer [22]. The discrepancy between the two studies was the result of an unequal distribution of methylation within the $M A L$ promoter, and ultimately the selection of more and less representative $\mathrm{CpG}$ sites that were included in the analyses [21].

An additional detail is the lack of standardization of commonly used methods which can also significantly contribute to diverging methylation frequencies reported for the same locus of interest, in the same samples and using the same technology [23]. By comparing more than 15,000 individual quantitative methylation-specific PCRs (qMSP; also called MethyLight [24]), the lack of proper normalization stood out as the most significant contributor to variability in the final DNA methylation results. Normalizing using robust elements such as the repetitive ALU element in contrast to single-/low-copy genes such as $A C T B$ has luckily become mainstream. However, the power of the normalization should not be overestimated. Large differences in DNA input among samples in the same study are one of many factors that will impact on the final methylation values [23].

Lack of standardization of the methylation analysis, in addition to inclusion of different $\mathrm{CpG}$ sites for analyses might also be a contributing factor to why the BLUEPRINT consortium recently recommended absolute quantitative analyses over qMSP/MethyLight [25]. Engaging 18 laboratories across 7 countries, they performed a quantitative comparison of the performance of the most widely used methods for DNA methylation analysis. Carefully selected, designed and validated qMSP/MethyLight assays were however acknowledged for the ability to detect small amounts of methylated DNA in an excess of unmethylated DNA in a cost-effective manner [25], in spite of the generally lower correlation between these assays compared with the absolute quantitative assays. Among the locus-specific analyses for DNA methylation assessment, qMSP/ MethyLight is commonly used. Overall, the method is fairly fast and simple, relatively cheap and has the potential for high-throughput by analyzing many samples in parallel. 


\section{Conclusion}

Only when including quality and standardization at every level of DNA methylation analyses, will we be able to achieve the robustness to independently validate DNA methylation analyses and to compare multiple methylation studies in systematic reviews. This is the only way to more efficiently develop future DNA methylation based biomarkers.

\section{References}

1 PubMed.

$$
\text { www.ncbi.nlm.nih.gov/pubmed }
$$

2 Jones PA. Functions of DNA methylation: islands, start sites, gene bodies and beyond. Nat. Rev. Genet. 13(7), 484-492 (2012).

3 Lei $\mathrm{H}, \mathrm{Oh}$ SP, Okano $\mathrm{M}$ et al. De novo DNA cytosine methyltransferase activities in mouse embryonic stem cells. Development 122(10), 3195-3205 (1996).

4 Okano M, Bell DW, Haber DA, Li E. DNA methyltransferases Dnmt3a and Dnmt3b are essential for de novo methylation and mammalian development. Cell 99(3), 247-257 (1999).

5 Zhong J, Agha G, Baccarelli AA. The role of DNA methylation in cardiovascular risk and disease: methodological aspects, study design, and data analysis for epidemiological studies. Circ. Res. 118(1), 119-131 (2016).

6 Sanchez-Mut JV, Heyn H, Vidal E et al. Human DNA methylomes of neurodegenerative diseases show common epigenomic patterns. Transl. Psychiatry 6, e718 (2016).

7 Barres R, Zierath JR. The role of diet and exercise in the transgenerational epigenetic landscape of T2DM. Nat. Rev Endocrinol. 12(8), 441-451 (2016).

8 Feinberg AP, Tycko B. The history of cancer epigenetics. Nat. Rev. Cancer 4(2), 143-153 (2004).

9 Schuebel KE, Chen W, Cope L et al. Comparing the DNA hypermethylome with gene mutations in human colorectal cancer. PLoS Genet. 3(9), 1709-1723 (2007).

10 Sahm F, Schrimpf D, Stichel D et al. DNA methylationbased classification and grading system for meningioma: a multicentre, retrospective analysis. Lancet Oncol. 18(5), 682-694 (2017).

11 Moran S, Martinez-Cardus A, Sayols S et al. Epigenetic profiling to classify cancer of unknown primary: a multicentre, retrospective analysis. Lancet Oncol. 17(10), 1386-1395 (2016).

12 Ioannidis JP, Bossuyt PM. Waste, leaks, and failures in the biomarker pipeline. Clin. Chem. 63(5), 963-972 (2017).

13 Umer M, Herceg Z. Deciphering the epigenetic code: an overview of DNA methylation analysis methods. Antioxid. Redox. Signal. 18(15), 1972-1986 (2013).

14 Havik AB, Brandal P, Honne $\mathrm{H}$ et al. MGMT promoter methylation in gliomas-assessment by pyrosequencing and

\section{Financial \& competing interests disclosure}

The authors have no relevant affiliations or financial involvement with any organization or entity with a financial interest in or financial conflict with the subject matter or materials discussed in the manuscript. This includes employment, consultancies, honoraria, stock ownership or options, expert testimony, grants or patents received or pending, or royalties.

No writing assistance was utilized in the production of this manuscript.

quantitative methylation-specific PCR. J. Transl. Med. 10, 36 (2012).

15 Weller M, van den BM, Hopkins K et al. EANO guideline for the diagnosis and treatment of anaplastic gliomas and glioblastoma. Lancet Oncol. 15(9), e395-e403 (2014).

16 Draht MX, Smits KM, Jooste V et al. Analysis of RET promoter $\mathrm{CpG}$ island methylation using methylation-specific PCR (MSP), pyrosequencing, and methylation-sensitive high-resolution melting (MS-HRM): impact on stage II colon cancer patient outcome. Clin. Epigenetics 8, 44 (2016).

17 Deckers IA, Schouten LJ, Van NL et al. Promoter methylation of CDO1 identifies clear-cell renal cell cancer patients with poor survival outcome. Clin. Cancer Res. 21(15), 3492-3500 (2015).

18 van VI, Joosten SC, De MT et al. A four-gene promoter methylation marker panel consisting of GREM1, NEURL, LAD1, and NEFH predicts survival of clear cell renal cell cancer patients. Clin. Cancer Res. 23(8), 2006-2018 (2017).

19 van VI, Niessen HE, Derks S et al. Analysis of promoter CpG island hypermethylation in cancer: location, location, location! Clin. Cancer Res. 17(13), 4225-4231 (2011).

20 Deng G, Chen A, Hong J, Chae HS, Kim YS. Methylation of $\mathrm{CpG}$ in a small region of the hMLH1 promoter invariably correlates with the absence of gene expression. Cancer Res. 59(9), 2029-2033 (1999).

21 Lind GE, Ahlquist T, Kolberg M et al. Hypermethylated MAL gene - a silent marker of early colon tumorigenesis. J. Transl. Med. 6(13), 13 (2008).

22 Mori Y, Cai K, Cheng Y et al. A genome-wide search identifies epigenetic silencing of somatostatin, tachykinin-1, and 5 other genes in colon cancer. Gastroenterology 131(3), 797-808 (2006).

23 Pharo HD, Honne H, Vedeld HM et al. Experimental factors affecting the robustness of DNA methylation analysis. Sci. Rep. 6, 33936 (2016).

24 Eads CA, Danenberg KD, Kawakami K et al. MethyLight: a high-throughput assay to measure DNA methylation. Nucleic Acids Res. 28(8), E32 (2000).

25 Bock C, Halbritter F, Carmona J et al. Quantitative comparison of DNA methylation assays for biomarker development and clinical applications. Nat. Biotechnol. 34(7), 726-737 (2016). 